\title{
Hybrid Pairs of Mappings with Some Weaker Conditions in Consideration of Common Fixed Point on 2-Metric Spaces
}

\author{
Bhavana Deshrande* And Rohit PathaK
}

\begin{abstract}
In this paper, we define weak commutativity of type $(K B)$ for hybrid pairs of mappings in 2-metric spaces. We prove a common fixed point theorem for two hybrid pairs of mappings by using weak commutativity of type $(K B)$ in 2-metric spaces. We give an example to throw light on our claim. We improve, extend and generalize several known results.
\end{abstract}

\section{INTRODUCTION}

Gähler $([29,30])$ introduced the concept of 2-metric spaces as a generalization of usual notion of metric space $(X, d)$. Gähler and many other mathematicians $([8,38,39])$ developed this notion extensively. The topology induced by 2-metric is called 2-metric topology which is generated by set of all open spheres with two centers. EL Naschie [21] used this sort of topology in physical applications. Many authors studied fixed point theorems in 2-metric spaces (Hsaio [1], Iseki [17]).

Gähler showed that the 2-metric is continuous in all of its three arguments but it need not be continuous in two arguments. A 2-metric which is continuous in all of its arguments is said to be continuous.

A new class of generalized metric space called D-metric space was introduced by Dhage [6] in 1992. Dhage attempted to develop topological structures in such spaces $([5,7,8])$. In 2003, Mustafa and Sims [39] proved that the most of the claims concerning the fundamental topological structures of D-metric spaces were incorrect.

Sessa [34] introduced the concept of weakly commuting maps. Jungck [13] defined the notion of compatible maps in order to generalize the concept of weak commutativity and showed that weakly commuting mappings are compatible but the converse is not true.

2010 Mathematics Subject Classification. Primary 54H25; Secondary 47H10.

Key words and phrases. Coincidence point, common fixed point, compatible maps, weak commutativity of type $(K B)$.

${ }^{*}$ Corresponding author. 
Jungck and Rhoades $([11,12])$ defined the concepts of $\delta$-compatibility and weak compatibility between a set valued mapping and a single-valued mapping and generalized the weak commutativity defined in [4]. Several authors used these concepts to prove some fixed point theorems [3, 9, 10, 2224]).

Monsef et al. [2] generalized some concepts in 2-metric spaces for set valued mappings. They also proved some common fixed point theorems in 2-metric spaces.

Fixed point theorems for set valued and single valued mappings provide technique for solving variety of applied problems in mathematical sciences and engineering (e.g., Krzyska and Kubiaczyk [32], Sessa and Khan [33]).

Pant ([25-28]) initiated the study of noncompatible maps and introduced pointwise R-weak commutativity of mappings in [27]. He also showed that pointwise R-weak commutativity is a necessary, hence minimal, condition for the existence of a common fixed point of contractive type maps [25]. In 1998, Jungck and Rhoades [11] also studied noncompatible maps and defined the concept of weak compatibility.

Pathak et al. [15] introduced the concept of R-weakly commuting maps of type $(A)$ and showed that they are not compatible.

Recently, Kubiaczyk and Deshpande [16] extended the concept of Rweakly commutativity of type $(A)$ for single valued mappings to set valued mappings and introduced weak commutativity of type $(K B)$ which is weaker condition than $\delta$-compatibility.

By using weak commutativity of type $(K B)$, Sharma and Deshpande [35] proved fixed point theorems for set and single valued noncompatible maps without continuity of any mappings. By using weak commutativity of type $(K B)$, Kubiaczyk and Deshpande [16] improved and generalized the results of Fisher [3], Khan et al. [20], Ahmad [18] and proved fixed point theorem for set and single valued noncompatible maps without assuming continuity of any mapping.

In this paper, we define the concept of weak commutativity of type $(K B)$ in 2-metric spaces and prove fixed point theorem using this concept. We improve and generalize the results of Fisher [3], Khan et al. [20], Ahmad [18]. We also generalize the result of Kubiaczyk and Deshpande [16].

\section{Preliminaries}

Definition 1 ([31]). Let $X$ denotes a nonempty set and $R$, the set of all nonnegative numbers. Then $X$ together with a function $d: X \times X \times X \rightarrow R$, is called a 2 -metric space if it satisfies the following properties:

(1) For distinct points $x, y \in X$, there exists a point $c \in X$ such that

$$
d(x, y, c) \neq 0 \text { and } d(x, y, c)=0
$$

if at least two of $x, y$ and $c$ are equal. 
(2) $d(x, y, c)=d(x, c, y)=d(y, x, c)=d(y, c, x)=d(c, x, y)=d(c, y, x)$. (Symmetry)

(3) $d(x, y, c) \leq d(x, y, z)+d(x, z, c)+d(z, y, c)$ for $x, y, c, z \in X$. (Rectangle inequality)

The function $d$ is called a $2-$ metric for the space $X$ and the pair $(X, d)$ denotes 2 -metric space. It has been shown by Gähler in [10] that 2-metric $d$ is non-negative and although $d$ is a continuous function of any of its three arguments, it need not be continuous in two arguments. A 2-metric $d$ which is continuous in all of its arguments is said to be continuous.

Geometrically, the value of a 2 -metric $d(x, y, c)$ represents the area of a triangle with vertices $x, y$ and $c$. Throughout this paper, let $(X, d)$ be a 2 -metric space unless mentioned otherwise and $B(X)$ is the class of all nonempty bounded subsets of $X$.

Definition 2 ([14]). A sequence $\left\{x_{n}\right\}$ in $(X, d)$ is said to be convergent to a point $x$ in $X$, denoted by $\lim _{n \rightarrow \infty} x_{n}=x$ if $\lim _{n \rightarrow \infty} d\left(x_{n}, x, c\right)=0$ for all $c$ in $X$. The point $x$ is called the limit of the sequence $\left\{x_{n}\right\}$ in $X$.

Definition 3 ([14]). A sequence $\left\{x_{n}\right\}$ in $(X, d)$ is said to be a Cauchy sequence if $\lim _{m, n \rightarrow \infty} d\left(x_{m}, x_{n}, c\right)=0$ for all $c$ in $X$.

Definition 4 ([14]). The space $(X, d)$ is said to be complete if every Cauchy sequence in $X$ converges to a point of $X$.

Remark 1. We note that, in a metric space a convergent sequence is a Cauchy sequence and in a 2 -metric space a convergent sequence need not be a Cauchy sequence, but every convergent sequence is a Cauchy sequence when the 2-metric $d$ is continuous on $X$ [24].

For all $A, B, C \in B(X)$, let $\delta(A, B, C)$ and $D(A, B, C)$ be the functions defined by

$$
\begin{aligned}
\delta(A, B, C) & =\sup \{d(a, b, c): a \in A, b \in B, c \in C\}, \\
D(A, B, C) & =\inf \{d(a, b, c): a \in A, b \in B, c \in C\} .
\end{aligned}
$$

If $A$ consists of a single point $a$ we write

$$
\delta(A, B, C)=\delta(a, B, C) .
$$

If $B$ and $C$ also consists of single points $b$ and $c$, respectively, we write

$$
\delta(A, B, C)=D(A, B, C)=d(a, b, c) .
$$

It follows immediately from the definition that

$$
\begin{aligned}
\delta(A, B, C) & =\delta(A, C, B)=\delta(C, B, A)=\delta(C, A, B)= \\
& =\delta(B, C, A)=\delta(B, A, C) \geq 0, \\
\delta(A, B, C) & \leq \delta(A, B, E)+\delta(A, E, C)+\delta(E, B, C),
\end{aligned}
$$


for all $A, B, C, E \in B(X)$ and $\delta(A, B, C)=0$ if at least two of $A, B$ and $C$ are singleton.

Definition $5([2])$. A sequence $\left\{A_{n}\right\}$ of subsets of a 2 -metric space $(X, d)$ is said to be convergent to a subset $A$ of $X$ if:

(i) given $a \in A$, there is a sequence $\left\{a_{n}\right\}$ in $X$ such that $a_{n} \in A_{n}$ for $n=1,2,3, \ldots$ and

$$
\lim _{n \rightarrow \infty} d\left(a_{n}, a, c\right)=0
$$

for all $c \in X$,

(ii) given $\varepsilon>0$, there exists a positive integer $N$ such that $A_{n} \subseteq A_{\varepsilon}$ for $n>N$ where $A_{\varepsilon}$ is the union of all open spheres with centers in $A$ and radius $\varepsilon$.

Definition 6 ([2]). The mappings $F: X \rightarrow B(X)$ and $f: X \rightarrow X$ are said to be weakly commuting on $X$ if $f F x \in B(X)$ and for all $C \in B(X)$

$$
\delta(F f x, f F x, C) \leq \max \{\delta(f x, F x, C), \delta(f F x, f F x, C)\} .
$$

Note that if $F$ is a single valued mapping, then the set $f F x$ consists of a single point. Therefore,

$$
\delta(f F x, f F x, C)=D(f F x, f F x, C)=0
$$

for all $C \in B(X)$ and the above inequality reduces to the condition given by Khan (19), that is

$$
D(F f x, f F x, C) \leq D(f x, F x, C) .
$$

Definition 7 ([2]). The mappings $F: X \rightarrow B(X)$ and $f: X \rightarrow X$ are said to be compatible if

$$
\lim _{n \rightarrow \infty} d\left(F f x_{n}, f F x_{n}, C\right)=0,
$$

whenever $\left\{x_{n}\right\}$ is a sequence in $X$ such that

$$
\lim _{n \rightarrow \infty} f x_{n}=t \in A=\lim _{n \rightarrow \infty} F x_{n}
$$

for some $t \in X$ and $A \in B(X)$.

Definition 8 ([2]). The mappings $F: X \rightarrow B(X)$ and $f: X \rightarrow X$ are said to be $\delta$-compatible if

$$
\lim _{n \rightarrow \infty} \delta\left(F f x_{n}, f F x_{n}, C\right)=0,
$$

whenever $\left\{x_{n}\right\}$ is a sequence in $X$ such that

$$
f F x_{n} \in B(X), F x_{n} \rightarrow\{t\} \quad \text { and } \quad f x_{n} \rightarrow t
$$

for some $t$ in $X$.

Definition 9 ([13]). The mappings $F: X \rightarrow B(X)$ and $f: X \rightarrow X$ are said to be weakly compatible if they commute at coincidence points, i.e., for each point $u$ in $X$ such that $F u=\{f u\}$, we have $F f u=f F u$. Note that the equation $F u=\{f u\}$ implies that $F u$ is a singleton. 
It can be easily shown that any $\delta$-compatible pair $\{F, f\}$ is weakly compatible but the converse is false.

Definition 10. The mappings $F: X \rightarrow B(X)$ and $f: X \rightarrow X$ are said to be weakly commuting of type $(K B)$ at $x$ if there exists some positive real number $R$ such that

$$
\delta(f f x, F f x, C) \leq R \delta(f x, F x, C)
$$

for all $C \in B(X)$.

Here $F$ and $f$ are weakly commuting of type $(K B)$ on $X$ if the above inequality holds for all $x \in X$.

Every $\delta$-compatible pair of hybrid maps is weakly commuting of type $(K B)$ but the converse is not necessarily true. For examples we can see $[16,35,36]$.

Lemma 1 ([2]). If $\left\{A_{n}\right\}$ and $\left\{B_{n}\right\}$ are sequences in $B(X)$ converging to $A$ and $B$ in $B(X)$, respectively, then the sequence $\left\{\delta\left(A_{n}, B_{n}, C\right)\right\}$ converges to $\delta(A, B, C)$.

Example 1. Let $X=[1,3]$ together with a function $d: X \times X \times X \rightarrow \mathbb{R}$ defined as

$$
d(x, y, z)=\min \{|x-y|,|y-z|,|z-x|\}
$$

for all $x, y, z \in X$.

Then $(X, d)$ is a 2-metric space. Define the mappings $I: X \rightarrow X$ and $F: X \rightarrow B(X)$ by

$$
I x=\left\{\begin{array}{ll}
x, & 1 \leq x \leq 2, \\
\frac{x+4}{3}, & 2<x \leq 3,
\end{array} \quad F x= \begin{cases}{[1, x],} & 1 \leq x \leq 2, \\
{[2, x],} & 2<x \leq 3 .\end{cases}\right.
$$

Let $x_{n}=2+\frac{1}{n}, n=1,2,3, \ldots$ Then

$$
\lim _{n \rightarrow \infty} I x_{n}=2 \text { and } \lim _{n \rightarrow \infty} F x_{n}=\{2\} .
$$

Also

$$
I F x_{n}=\left[2,2+\frac{1}{3 n}\right] \in B(X)
$$

and

$$
\lim _{n \rightarrow \infty} \delta\left(F I x_{n}, I F x_{n}, C\right)=\lim _{n \rightarrow \infty} \delta\left(\left[2,2+\frac{1}{3 n}\right],\left[2,2+\frac{1}{3 n}\right], C\right)=0 .
$$

Hence $I$ and $F$ are $\delta$-compatible.

If we take $x=2$, then $I I x=2$ and $F I x=[1,2]$. Therefore

$$
\delta(I I x, F I x, C)=\delta(2,[1,2], C) \leq R \delta(I x, F x, C)=\delta(2,[1,2], C)
$$

for all $R \geq 1$ and $C \in B(X)$.

Hence $F$ and $I$ are weakly commuting of type $(K B)$ at $x=2$. 
Example 2. Let $X=[0,4]$ together with a function $d: X \times X \times X \rightarrow \mathbb{R}$ defined as

$$
d(x, y, z)=\min \{|x-y|,|y-z|,|z-x|\}
$$

for all $x, y, z \in X$.

Then $(X, d)$ is a 2-metric space. Define the mappings $I: X \rightarrow X$ and $F: X \rightarrow B(X)$ by

$$
I x=\frac{x}{2} \text { and } F x=[1, x]
$$

for all $x \in X$.

Then

$$
I I x=\frac{x}{4} \text { and } F I x=\left[1, \frac{x}{2}\right] .
$$

Therefore

$$
\delta(I I x, F I x, C)=\delta\left(\frac{x}{4},\left[1, \frac{x}{2}\right], C\right) \leq R \delta(I x, F x, C)=R \delta\left(\frac{x}{4},[1, x], C\right)
$$

for all $R \geq 1$ and $C \in B(X)$.

Thus $I$ and $F$ are weakly commuting of type $(K B)$ on $X$ but there exists no sequence in $X$ such that the condition of $\delta$-compatibility is satisfied.

\section{Main Results}

Theorem 11. Let $(X, d)$ be a 2 -metric space and $I, J: X \rightarrow X$ be self mappings and $F, G: X \rightarrow B(X)$ be set valued mappings such that:

(1.1) $\cup G(X) \subseteq I(X)$ and $\cup F(X) \subseteq J(X)$,

$$
\delta(F x, G y, C) \leq \max \left\{\begin{array}{c}
c D(I x, J y, C), c \delta(I x, F x, C), c \delta(J y, G y, C), \\
a D(I x, G y, C)+b D(J y, F x, C)
\end{array}\right\}
$$

for all $x, y \in X$ and $C \in B(X)$ where

$$
0 \leq c<1, a \geq 0, b \geq 0, a+b<1, c \max \left\{\frac{a}{1-a}, \frac{b}{1-b}\right\}<1,
$$

(1.3) one of $I(X)$ or $J(X)$ is complete,

(1.4) the pairs $\{F, I\}$ and $\{G, J\}$ are weakly commuting of type $(K B)$ at coincidence points in $X$.

Then there exists a unique fixed point $z$ in $X$ such that

$$
\{z\}=\{I z\}=\{J z\}=F z=G z .
$$

Proof. Let $x_{0} \in X$ be an arbitrary point in $X$. By (1.1), there exists a point $x_{1}$ in $X$ such that $J x_{1} \in F x_{0}=Z_{0}$ and for this point $x_{1}$ there exists a point $x_{2}$ in $X$ such that $I x_{2} \in G x_{1}=Z_{1}$ and so on. Continuing in this manner, we can define a sequence $\left\{x_{n}\right\}$ as follows:

$$
J x_{2 n+1} \in F x_{2 n}=Z_{2 n}, I x_{2 n+2} \in G x_{2 n+1}=Z_{2 n+1}, \quad \text { for } n=0,1,2,3, \ldots
$$


Let $V_{n}=\delta\left(Z_{n}, Z_{n+1}, C\right)$ for $n=0,1,2,3, \ldots$ By (1.2), we have

$$
\begin{aligned}
V_{2 n} & =\delta\left(Z_{2 n}, Z_{2 n+1}, C\right) \\
& =\delta\left(F x_{2 n}, G x_{2 n+1}, C\right) \\
& \leq \max \left\{\begin{array}{c}
c D\left(I x_{2 n}, J x_{2 n+1}, C\right), c \delta\left(I x_{2 n}, F x_{2 n}, C\right), \\
c \delta\left(J x_{2 n+1}, G x_{2 n+1}, C\right), \\
a D\left(I x_{2 n}, G x_{2 n+1}, C\right)+b D\left(J x_{2 n+1}, F x_{2 n}, C\right)
\end{array}\right\} \\
& \leq \max \left\{c V_{2 n-1}, c V_{2 n}, a\left(V_{2 n-1}+V_{2 n}\right)\right\} \\
& \leq V_{2 n-1} \max \left\{c, \frac{a}{1-a}\right\}
\end{aligned}
$$

for $n \in N$.

Similarly, one can show that

$$
\begin{aligned}
V_{2 n+1} & =\delta\left(Z_{2 n+1}, Z_{2 n+2}, C\right) \\
& =\delta\left(G x_{2 n+1}, F x_{2 n+2}, C\right) \\
& \leq V_{2 n} \max \left\{c, \frac{b}{1-b}\right\}
\end{aligned}
$$

for $n \in N$.

If we put

$$
\beta=\max \left\{c, \frac{a}{1-a}\right\} \cdot \max \left\{c, \frac{b}{1-b}\right\},
$$

then by hypothesis it can be easily seen that $0 \leq \beta<1$. So we deduce that

$$
\begin{aligned}
V_{2 n} & \leq \beta V_{2 n-2} \leq \cdots \leq \beta^{n} V_{0}, \\
V_{2 n+1} & \leq \beta V_{2 n-1} \leq \cdots \leq \beta^{n} V_{1},
\end{aligned}
$$

for $n \in N$.

Put $M=\max \left\{V_{0}, V_{1}\right\}$. It follows from the above inequality that if $z_{n}$ is an arbitrary point in the set $Z_{n}$ for $n \in N$, then we obtain that

$$
D\left(z_{2 n}, z_{2 n+1}, C\right) \leq \delta\left(Z_{2 n}, Z_{2 n+1}, C\right) \leq \beta^{n} M
$$

and

$$
D\left(z_{2 n+1}, z_{2 n+1}, C\right) \leq \delta\left(Z_{2 n+1}, Z_{2 n+2}, C\right) \leq \beta^{n} M .
$$

This implies that $\left\{z_{n}\right\}$ and hence any subsequence thereof, is a Cauchy sequence in $X$.

Now suppose that $J(X)$ is complete, since $J x_{2 n+1} \in F x_{2 n}=Z_{2 n}$ for $n=0,1,2, \ldots$

$$
D\left(J x_{2 m+1}, J x_{2 n+1}, C\right) \leq \delta\left(Z_{2 m}, Z_{2 n}, C\right)<\varepsilon
$$

for $m, n \geq n_{0}, n_{0}=1,2,3, \ldots$

Therefore $\left\{J x_{2 n+1}\right\}$ is a Cauchy sequence and hence $J x_{2 n+1} \rightarrow z=J v \in$ $J(X)$. But $I x_{2 n} \in G x_{2 n-1}=Z_{2 n-1}$ and whence, we have

$$
D\left(I x_{2 n}, J x_{2 n+1}, C\right) \leq \delta\left(Z_{2 n-1}, Z_{2 n}, C\right)=V_{2 n-1} \rightarrow 0 .
$$


Consequently, $I x_{2 n} \rightarrow z$. Moreover, we have for $n=1,2,3, \ldots$

$$
\begin{aligned}
\delta\left(F x_{2 n}, z, C\right) & \leq \delta\left(F x_{2 n}, I x_{2 n}, C\right)+\delta\left(I x_{2 n}, z, C\right) \\
& \leq \delta\left(Z_{2 n}, Z_{2 n-1}, C\right)+d\left(I x_{2 n}, z, C\right) .
\end{aligned}
$$

Therefore $\delta\left(F x_{2 n}, z, C\right) \rightarrow 0$.

Similarly $\delta\left(G x_{2 n-1}, z, C\right) \rightarrow 0$. For $n=1,2,3, \ldots$ by $(1.2)$ we have

$$
\begin{aligned}
\delta\left(F x_{2 n}, G v, C\right) & \leq \max \left\{\begin{array}{c}
c D\left(I x_{2 n}, J v, C\right), c \delta\left(I x_{2 n}, F x_{2 n}, C\right), c \delta(J v, G v, C), \\
a D\left(I x_{2 n}, G v, C\right)+b D\left(J v, F x_{2 n}, C\right)
\end{array}\right\} \\
& \leq \max \left\{\begin{array}{c}
c D\left(I x_{2 n}, J v, C\right), c \delta\left(I x_{2 n}, F x_{2 n}, C\right), \\
c \delta(J v, G v, C), \\
a \delta\left(I x_{2 n}, G v, C\right)+b \delta\left(J v, F x_{2 n}, C\right)
\end{array}\right\}
\end{aligned}
$$

and since $\delta\left(I x_{2 n}, G v, C\right) \rightarrow \delta(z, G v, C)$, when $I x_{2 n} \rightarrow z$, we get as $n \rightarrow \infty$

$$
\delta(z, G v, C) \leq \max \{c, a\} \delta(z, G v, C),
$$

which is a contradiction. Thus $G v=\{z\}=\{J v\}$. But $\cup G(X) \subseteq I(X)$, there exists $u \in X$ such that $\{I u\}=G v=\{J v\}=\{z\}$.

Now if $F u \neq G v, \delta(F u, G v, C) \neq 0$, then by (1.2), we have

$$
\begin{aligned}
\delta(F u, G v, C) & \leq \max \left\{\begin{array}{c}
c D(I u, J v, C), c \delta(I u, F u, C), c \delta(J v, G v, C), \\
a D(I u, G v, C)+b D(J v, F u, C)
\end{array}\right\} \\
& \leq \max \left\{\begin{array}{c}
c D(I u, J v, C), c \delta(I u, F u, C), c \delta(J v, G v, C), \\
a \delta(I u, G v, C)+b \delta(J v, F u, C) .
\end{array}\right\}
\end{aligned}
$$

Hence, we have $\delta(F u, G v, C) \leq \max \{c, b\} \delta(F u, G v, C)$. This is a contradiction. Thus we have

$$
F u=\{I u\}=\{J v\}=G v=\{z\} .
$$

Since $F u=\{I u\}$ and the pair $\{F, I\}$ is weakly commuting of type $(K B)$ at coincidence points in $X$, we obtain

$$
\delta(I I u, F I u, C) \leq R \delta(I u, F u, C)
$$

which gives $\{I z\}=F z$.

Again since $G v=\{J v\}$ and the pair $\{G, J\}$ is weakly commuting of type $(K B)$ at coincidence points in $X$, we obtain

$$
\delta(J J v, G J v, C) \leq R \delta(J v, G v, C)
$$

which gives $\{J z\}=G z$.

By (1.2), we have

$$
\begin{aligned}
\delta(F z, z, C) & \leq \delta(F z, G v, C) \\
& \leq \max \left\{\begin{array}{c}
c D(I z, J v, C), c \delta(I z, F z, C), c \delta(J v, G v, C), \\
a D(I z, G v, C)+b D(J v, F z, C)
\end{array}\right\} \\
& \leq \max \{c, a+b\} \delta(F z, z, C) .
\end{aligned}
$$


Here we reach a contradiction. Thus $F z=\{z\}$. Consequently, we have

$$
\{z\}=F z=\{I z\} .
$$

Similarly

$$
\{z\}=G z=\{J z\}
$$

Therefore, we have

$$
\{z\}=\{I z\}=\{J z\}=F z=G z .
$$

Finally, we prove that $z$ is unique. If not then we let $w$ to be another common fixed point such that $z \neq w$ and

$$
\{w\}=\{I w\}=\{J w\}=F w=G w .
$$

By (1.2), we have

$$
\begin{aligned}
D(z, w, C) & \leq \delta(F z, G w, C) \\
& \leq \max \left\{\begin{array}{c}
c D(I z, J w, C), c \delta(I z, F z, C), c \delta(J w, G w, C), \\
a D(I z, G w, C)+b D(J w, F z, C)
\end{array}\right\} \\
& \leq \max \{c, a+b\} D(z, w, C),
\end{aligned}
$$

which is a contradiction. Therefore $z=w$. This completes the proof.

Example 3. Let $X=[0,2)$ together with a function $d: X \times X \times X \rightarrow \mathbb{R}$ defined as

$$
d(x, y, z)=\min \{|x-y|,|y-z|,|z-x|\}
$$

for all $x, y, z \in X$. Then $(X, d)$ is a 2 -metric space.

Define the mappings

$$
I: X \rightarrow X, J: X \rightarrow X \text { and } F: X \rightarrow B(X), G: X \rightarrow B(X)
$$

by

$$
\begin{gathered}
I x=\left\{\begin{array}{ll}
2 x, & 0 \leq x \leq 1, \\
x, & 1<x \leq 2,
\end{array} \quad \text { Jx }= \begin{cases}x, & 0 \leq x \leq 1, \\
\frac{x}{2}, & 1<x \leq 2\end{cases} \right. \\
F x=\left\{\begin{array}{ll}
{\left[0, \frac{x}{2}\right],} & 0 \leq x \leq 1, \\
{\left[0, \frac{x}{4}\right],} & 1<x \leq 2,
\end{array} \quad G x= \begin{cases}{[0, x],} & 0 \leq x \leq 1, \\
{\left[0, \frac{x}{2}\right],} & 1<x \leq 2 .\end{cases} \right.
\end{gathered}
$$

Then

$$
\begin{aligned}
& \text { 1. } \cup G(X) \subseteq I(X) \text { and } \cup F(X) \subseteq J(X), \\
& \text { 2. for } c=1 / 2, a=1 / 5, b=1 / 4 \\
& \delta(F x, G y, C) \leq \max \left\{\begin{array}{c}
c D(I x, J y, C), c \delta(I x, F x, C), c \delta(J y, G y, C), \\
a D(I x, G y, C)+b D(J y, F x, C)
\end{array}\right\}
\end{aligned}
$$

3. The pairs $\{F, I\}$ and $\{G, J\}$ are weakly commuting of type $(K B)$ at the coincidence point $x=0$.

Therefore all the conditions of the Theorem 1 are satisfied. The common fixed point is $x=0$. 
Now if we put $F=G$ and $I=J$ in Theorem 1, we obtain the following Corollary.

Corollary 1. Let $(X, d)$ be a 2 -metric space and $I: X \rightarrow X$ be self mapping and $F: X \rightarrow B(X)$ be set valued mapping such that:

(2.1) $\cup F(X) \subseteq I(X)$,

(2.2) $\delta(F x, F y, C) \leq \max \left\{\begin{array}{c}c D(I x, I y, C), c \delta(I x, F x, C), c \delta(I y, F y, C), \\ a D(I x, F y, C)+b D(\operatorname{Iy}, F x, C)\end{array}\right\}$ for all $x, y \in X$ and $C \in B(X)$ where

$0 \leq c<1, a \geq 0, b \geq 0, a+b<1, c \max \left\{\frac{a}{1-a}, \frac{b}{1-b}\right\}<1$,

(2.3) $I(X)$ is complete,

(2.4) the pair $\{F, I\}$ is weakly commuting of type $(K B)$ at coincidence points in $X$.

Then there exists a unique fixed point $z$ in $X$ such that

$$
\{z\}=\{I z\}=F z .
$$

\section{REFERENCES}

[1] A. Hsiao, Property of contractive type mappings in 2-metric spaces, Thanabha 16 (1986), 223-39.

[2] Abd EL-Monsef, H.M. Abu-Donia, Abd-Rabou Kh., New types of common fixed point theorems in 2-metric spaces, Chaos Solitons and Fractals 41 (2009), 1435-1441.

[3] B. Fisher, Common fixed points of mappings and set-valued mappings on metric spaces, Kyungpook Math. J., 25 (1985), 35-42.

[4] B. Fisher, S. Sessa, Two common fixed point theorems for weakly commuting mappings, Periodica Math Hungarica, 20 (3) (1989), 207-218.

[5] B.C. Dhage, On generalized metric spaces and topological structure II, Pure and Applied Mathematika Sciences, 40 (1-2) (1994), 37-41.

[6] B.C. Dhage, Generalized metric space and mapping with fixed point, Bulletin of Calcutta Mathematical Society, 84 (6) (1992), 329-334.

[7] B.C. Dhage, Generalized metric spaces and topological structure I, Analele Stiintifice ale Universitatii Al. I. Cuza Din Iasi Serie Noud. Matematica, 46(1) (2000), 3-24.

[8] B.C. Dhage, On continuity of mappings in D-metric spaces, Bulletin of Culcutta Mathematical Society, 86(6) (1994), 503-508.

[9] B.E. Rhoades, Common fixed points of compatible set-valued mappings, Publ Math Debrecen, 48(3-4) (1996), 237-40.

[10] B.E. Rhoades, S. Park, K.B. Moon, On generalizations of Meir-Keeler type contraction maps, J. Math. Anal. Appl., 146 (1990), 482.

[11] G. Jungck, B.E. Rhoades, Fixed points for set valued functions without continuity, Indian J. Pure and Appl. Math., 29 (1998), 227-38.

[12] G. Jungck, B.E. Rhoades, Some fixed point theorems for compatible maps, Int. J. Math. Sci., 16 (1993), 417-28. 
[13] G. Jungck, Compatible mappings and common fixed points, Int. J. Math. Sci., 9 (1986), 771-779.

[14] H.K. Pathak, S.M. Kang, J.H. Baek, Weak compatible mappings of type $(A)$ and common fixed points, Kyungpook Math. J., 35 (1995), 345-59.

[15] H.K. Pathak, Y.J. Cho Kang, Remarks on R-weakly commuting mappings and common fixed point theorems, Bull. Korean Math. Soc., 34 (1997), 247-257.

[16] I. Kubiaczyk, B. Deshpande, Noncompatibility, Discontinuity in consideration of common fixed point of set and single-valued maps, Southeast Asian Bull. of Math., 32 (2008), 467-474.

[17] K. Iseki, Fixed point theorems in 2-metric spaces, Math. Sem. notes, 3 (1975), 133-6.

[18] M.A. Ahmed, Common fixed point theorems for set and single valued mappings, Demonstratio Mathematica, XXXVI, 3 (2003), 471-481.

[19] M.D. Khan, A study of fixed point theorems, Ph.D. thesis, Aligarah Muslim University, 1984.

[20] M.S. Khan, I. Kubiaczyk, S. Sessa, On set valued and single valued mappings with common fixed points, Bull. Malaysia Math. Soc., 2(10) (1987), 71-81.

[21] MS.El. Naschie, Wild topology hyperbolic geometry and fusion algebra of high energy particle physics, Chaos, Solitons \& Fractals, 13 (2002), 1935-45.

[22] R.A. Rashwan, Fixed points of single and set-valued mappings, Kyungpook Math. J., 38 (1998), 29-37.

[23] R.A. Rashwan, M.A. Ahmed, Common fixed points for weakly compatible mappings, Italian J. of Pure and Appl. Math., 8 (2000), 35-44.

[24] R.A. Rashwan, M.A. Ahmed, Common fixed points for $\delta$-compatible mappings, Southwest J. Pure Appl. Math., 1 (1996), 51-61.

[25] R.P. Pant, Common fixed point theorems for contractive maps, J. Math. Anal. Appl., 226 (1998), 251-258.

[26] R.P. Pant, Common fixed points of Lipschitz type mapping pair, J. Math. Anal. Appl., 240 (1999), 280-283.

[27] R.P. Pant, Common fixed points of noncommuting mappings, J. Math. Anal. Appl., 188 (1994), 436-440.

[28] R.P. Pant, Discontinuity and fixed points, J. Math. Anal. Appl., 240 (1999), 284-289.

[29] S. Gähler, Zur geometric 2-metrische Raume, Rev. Raum. Math. Pures et Appl., 11 (1966), 655-64.

[30] S. Gähler, 2-metrische Raume und ihre topologische structure, Math. Nacher., 26 (1963), 115-48.

[31] S. Gähler, Uber die niformisierbakait 2-metrische Raume, Math. Nacher., 28 (1965), 235-44.

[32] S. Krzyska, I. Kubiaczyk, Fixed point theorems for upper semi continuous and weaklyweakly upper semi continuous multivalued mappings, MathJaponica, 47(2) (1998), 237-240.

[33] S. Sessa, M.S. Khan, Some remarks in best approximation theory, Math. J. Toyoma Univ., 17 (1994), 151-165. 
[34] S. Sessa, On weak commutativity condition of mappings in fixed point cosiderations, Publ. Inst. Math. (Beograd), 32(46) (1982), 149-153.

[35] S. Sharma, B. Deshpande, Fixed point theorems for set and single valued maps without continuity and compatibility, Demonstratio Mathematica, Vol. XL 3 (2007), 649-658.

[36] S. Sharma, B. Deshpande, R. Pathak, Common fixed point theorems for hybrid pairs of mappings with some weaker conditions, Fasciculi Math., 39 (2008), 53-67.

[37] S.V.R. Naidu, J.R. Prasad, Fixed point theorem in 2-metric spaces, Indian J. Pure and Appl. Math., 17 (1986), 974-93.

[38] Z. Mustafa, U. Sims, A new approach to generalized metric spaces, Journal of Nonlinear and Convex Analysis, 7(2) (2006), 289-297.

[39] Z. Mustafa, U. Sims, Some remarks concerning D-metric spaces, International Conference of Fixed point Theory and Applications, Yokohama, 2004, 189-198.

\section{Bhavana Deshrande}

Govt. Arts and Science P.G. College

RATLAM (M.P.)

INDIA

E-mail address: bhavnadeshpande@yahoo.com

\section{Rohit PAthak}

Institute of Engineering And TeChnology DAVV, INDORE (M.P.)

INDIA

E-mail address: rohitpathakres@yahoo.in 